\title{
Pacific
}

Journal of

Mathematics

\section{ON CLOSED HYPERSURFACES OF CONSTANT SCALAR CURVATURES AND MEAN CURVATURES IN $S^{n+1}$}

\author{
SHAOPING CHANG
}




\title{
ON CLOSED HYPERSURFACES OF CONSTANT SCALAR CURVATURES AND MEAN CURVATURES IN $S^{n+1}$
}

\section{ShaOPING Chang}

\begin{abstract}
We consider in this note the following question: given a closed Riemann $n$-manifold of constant scalar curvature, how can it be minimally immersed in the round $(n+1)$-sphere? Our main result states that the immersion has to be isoparametric if the number of its distinct principal curvatures is three identically. This provides another piece of supporting evidence to a conjecture of Chern.
\end{abstract}

0. Introduction. Consider $\mathscr{M}_{\text {closed }}^{n}$ the set of all the closed minimal hypersurfaces of constant scalar curvatures $R$ in the unit round $(n+1)$-sphere $S^{n+1}$. Let $\mathscr{R}_{n} \subset \mathbf{R}$ be the collection of all the possible values of such $R$ 's. Chern [12] posed the following:

Chern Conjecture. For any $n \geq 3, \mathscr{R}_{n}$ is a discrete subset of the real numbers.

This is a very interesting conjecture in the theory of minimal submanifolds in spheres. To attack this problem, it will be most helpful if one has a good guess on what $\mathscr{M}_{\text {closed }}^{n}$ is for each $n$. When $n=3$, from his work on the exterior differential systems R. Bryant [1] proposed the following:

Bryant Conjecture. A piece of minimal hypersurface of constant scalar curvature in $S^{4}$ is isoparametric of type $g \leq 3$.

Here a hypersurface (not necessarily compact) $M^{n}$ in $S^{n+1}$ is said to be isoparametric of type $g$ if it has constant principal curvatures $\lambda_{1}<\cdots<\lambda_{g}$ with respective constant multiplicities $m_{1}, \ldots, m_{g}$. Such hypersurfaces with $g \leq 3$ are classified due to Cartan's work [2] in 1939.

Note that the Bryant conjecture is very strong because $M^{3}$ is not assumed to be closed. Nevertheless, there is good evidence that it may be true. In [3], together with the works of Simons [11] and Peng-Terng [10], the author was able to establish the Chern Conjecture when $n=3$ by showing that each $M^{3} \in \mathscr{M}_{\text {closed }}^{3}$ is an isoparametric hypersurface. Hence, $\mathscr{R}_{3}=\{0,3,6\}$. Also, the Bryant Conjecture was verified 
when $M^{3}$ has multiple principal curvatures somewhere.

Therefore, we would like to pursue such a point of view for the study of $\mathscr{M}_{\text {closed }}^{n}$ in higher dimensions. Suppose that $M^{n}$ also satisfies the following:

Condition $(g)$ : The number $g$ of distinct principal curvatures is constant.

Recall that there is one minimal hypersurface among each family of isoparametric hypersurfaces (cf. [9]). All the closed minimal isoparametric hypersurfaces by definition are members of $\mathscr{M}_{\text {closed }}^{n}$ and satisfy Condition $(g)$. Conversely, it is straightforward to check that any $M^{n} \in \mathscr{M}_{\text {closed }}^{n}$ satisfying Condition ( $g$ ) with $g \leq 2$ has constant principal curvatures and thus is isoparametric. When $g=3$, as a consequence of the main result of the present paper, one has the following:

Theorem. If $M^{n} \in \mathscr{M}_{\text {closed }}^{n}$ satisfies Condition ( $g$ ) with $g=3$, then $M^{n}$ is either an equator $S^{n}$, a product of spheres $S^{p} \times S^{q}$ or a Cartan minimal hypersurface.

REMARK. The Bryant conjecture will be established if one can exhibit such a theorem without assuming $M^{n}$ to be compact.

We now state the following:

MaIn Theorem. A closed hypersurface $M^{n}$ of constant scalar curvature $R$ and constant mean curvature $H$ in $S^{n+1}$ is isoparametric provided it has 3 distinct principal curvatures everywhere.

REMARK. When the principal curvatures are all non-simple, $R$. Miyaoka [7] exhibited that $M^{n}$ is isoparametric even without assuming the scalar curvature is constant.

Acknowledgment. We wish to express our thanks to Dr. Y. Xu for his interest in the work and to Professor S. Y. Cheng for his valuable comments and continuous support and encouragement.

1. Notations and the reduction of the proof. Throughout the paper, we use $A, B, C, \ldots$, for indices ranging from 1 to $n$ and denote by $\delta_{A B}$ the Kronecker symbols. 
For each point $x \in M^{n}$, let $\lambda(x), \mu(x)$ and $\sigma(x)$ be the three distinct principal curvatures of multiplicities $p(x), q(x)$ and $r(x)$, respectively, at $x$.

In order to establish the Main Theorem, we need to show that all the three continuous functions $\lambda, \mu$ and $\sigma$ on $M^{n}$ are indeed constant functions.

We first observe that all the three integer-valued functions $p, q$ and $r$ are constant integers.

Indeed, consider the following system of linear equations with $p$, $q$ and $r$ as unknowns:

$$
\begin{aligned}
p+q+r & =n, \\
p \lambda+q \mu+r \sigma & =H, \\
p \lambda^{2}+q \mu^{2}+r \sigma^{2} & =S,
\end{aligned}
$$

where $S$ is the square length of the second fundamental form.

Since $\lambda, \mu$ and $\sigma$ are distinct everywhere, we can solve for $p, q$ and $r$ in terms of $\lambda, \mu, \sigma$ and $S$, which are all continuous on $M^{n}$.

This shows that $p, q$ and $r$ are constant as desired since they need to be integers.

Remark. By the same argument, one can see that for $\forall g$, Condition $(g)$ always yields the constancy of the multiplicities.

Therefore, we can choose a local frame $\left\{e_{i}, e_{\alpha}, e_{a}\right\}$ where the indices $i, \alpha$ and $a$ range from 1 to $p, p+1$ to $p+q$ and $p+q+1$ to $p+q+r(=n)$, respectively, such that the second fundamental form $h=\sum_{A, B} h_{A B} \omega_{A} \omega_{B}$ is given by

$$
\left(h_{A B}\right)=\left(\begin{array}{lll}
\lambda I_{p} & & \\
& \mu I_{q} & \\
& & \sigma I_{r}
\end{array}\right)
$$

where for each integer $s$, we denote by $I_{s}$ the identity matrix of rank $s$, and $\left\{\omega_{i}, \omega_{\alpha}, \omega_{a}\right\}$ is the dual co-frame of $\left\{e_{i}, e_{\alpha}, e_{a}\right\}$.

Recall that the structure equations of $M^{n}$ are given by the following:

$$
\begin{aligned}
d \omega_{A} & =\sum_{B} \omega_{A B} \wedge \omega_{B}, \\
d \omega_{A B} & =\sum_{C} \omega_{A C} \wedge \omega_{C B}-\frac{1}{2} \sum_{C, D} R_{A B C D} \omega_{C} \wedge \omega_{D},
\end{aligned}
$$

where $\omega_{A B}$ 's denote the connection forms of $M^{n}$ and $R_{A B C D}$ the curvature tensor. 

by

Define $\nabla h=\sum_{A, B, C} h_{A B C} \omega_{A} \omega_{B} \omega_{C}$ the covariant derivative of $h$ $(\nabla h)$

$$
\sum_{C} h_{A B C} \omega_{C}=d h_{A B}+\sum_{C} h_{C B} \omega_{C A}+\sum_{C} h_{A C} \omega_{C B}
$$

Then, by virtue of $(h),(\nabla h)$ can be interpreted as

$$
\begin{aligned}
& \sum_{C} h_{i j C} \omega_{C}=\delta_{i j} d \lambda, \\
& \sum_{C} h_{\alpha \beta C} \omega_{C}=\delta_{\alpha \beta} d \mu, \\
& \sum_{C} h_{a b C} \omega_{C}=\delta_{a b} d \sigma, \\
& \sum_{C} h_{i \alpha C} \omega_{C}=(\lambda-\mu) \omega_{i \alpha}, \\
& \sum_{C} h_{i n C} \omega_{C}=(\lambda-\sigma) \omega_{i n}, \\
& \sum_{C} h_{\alpha a C} \omega_{C}=(\mu-\sigma) \omega_{\alpha a} .
\end{aligned}
$$

Recall that $h_{A B C}$ is symmetric in all the indices since the ambient space $S^{n+1}$ is of constant curvature and (cf. [4])

$$
\sum_{A, B, C} h_{A B C}^{2}=S(S-n)+H^{2}-H f
$$

where $f=\sum_{A, B, C} h_{A B} h_{B C} h_{C A}$.

Note that $S=n(n-1)+H^{2}-R$ (cf. [4]) is constant and all the principal curvatures $\lambda, \mu$ and $\sigma$ are smooth functions on $M^{n}$.

By differentiating both $(*)$ and $f=p \lambda^{3}+q \mu^{3}+r \sigma^{3}$, we have

$$
\begin{aligned}
p d \lambda+q d \mu+r d \sigma & =0, \\
p \lambda d \lambda+q \mu d \mu+r \sigma d \sigma & =0, \\
p \lambda^{2} d \lambda+q \mu^{2} d \mu+r \sigma^{2} d \sigma & =\frac{1}{3} d f .
\end{aligned}
$$

It follows that

$$
\frac{p d \lambda}{\sigma-\mu}=\frac{q d \mu}{\lambda-\sigma}=\frac{r d \sigma}{\mu-\lambda}=\frac{d f}{3 D}
$$

where $D=(\sigma-\mu)(\sigma-\lambda)(\mu-\lambda)$. 
In the case when all the principal curvatures are non-simple, from the Miyaoka theorem [7], we immediately assert that $M^{n}$ is isoparametric.

And the case when $p=q=r=1$ was already verified by the author in [4]. It therefore suffices to show that all the principal curvatures are simple if so is one of them, say, $r=1$.

To this aim, we need the following:

KeY LEMMA. With the same notations as above. If $r=1$ and $p q \geq 2$, then $h_{i \alpha n}=0, \forall i, \alpha$.

The proof of this lemma itself will be given in $\S 2$. We will finish the current section by showing how to achieve our aim from the Key Lemma.

Consider a point $x_{0} \in M^{n}$ where $d f=0$, from (\#) we have

$$
\begin{aligned}
d \lambda & =d \mu=d \sigma=0 \\
\text { i.e. } \quad h_{i j A} & =h_{\alpha \beta A}=h_{a b A}=0, \quad \forall i, j, \alpha, \beta, a, b, A .
\end{aligned}
$$

Now suppose otherwise that $r=1$ and $p q \geq 2$.

From the Key Lemma, the left-hand side of $(S)$ would vanish at $x_{0}$ and then

$$
S(S-n)+H^{2}-H f\left(x_{0}\right)=0 .
$$

When $H \neq 0$, since $d f=0$ at both maximum and minimum points of $f$, it would follow that $f=\frac{1}{H}\left(S(S-n)+H^{2}\right)$ identically. From (\#), this in turn would yield that $\lambda, \mu$ and $\sigma$ were constant and then $M^{n}$ be isoparametric, contradicting the classification by Cartan.

When $H=0$, it would follow that $S(S-n)=0$ and then $M^{n}$ be either an equator or a product of spheres, due to Chern-do CarmoKobayashi and Lawson $[5,6]$, contradicting the assumption that $g=$ 3 .

2. Proof of the Key Lemma. At each point $x \in M^{n}$, denote by $Y$ the $p \times q$ matrix $\left(h_{i \alpha n}\right) \in M_{p \times q}$. We are supposed to show that $Y=0$ everywhere if $r=1$ and $p q \geq 2$.

We will employ the following [8]:

THEOREM [Otsuki, 1970]. Let $M^{n}$ be a hypersurface immersed in an $(n+1)$-dimensional Riemannian manifold of constant curvature such that the multiplicities of principal curvatures are all constant.

Then the distribution of the space of principal vectors corresponding to each principal curvature is completely integrable. Moreover, if the 
multiplicity of a principal curvature is greater than 1 , then this principal curvature is constant on each integral submanifold of the corresponding distribution of the space of principal vectors.

Now, without loss of generality, assume that $q \geq 2$.

Applying the Otsuki theorem to $\mu$ and noting that $d \lambda=\frac{\mu-\sigma}{\sigma-\lambda} d \mu$ and $d \sigma=\frac{\mu-\lambda}{\lambda-\sigma} d \mu$ from (\#), we have

$$
\lambda_{\alpha}=\mu_{\alpha}=\sigma_{\alpha}=0, \quad \forall \alpha .
$$

Case 1. $p=1$.

Rewrite $(1.1) \rightarrow(2.3)$ as

(I.1) $\quad h_{11 C}=\lambda_{C}, \quad h_{\alpha \alpha C}=\mu_{C}, \quad h_{n n C}=\sigma_{C}, \quad \forall \alpha, C$,

(I.2) $\quad h_{\alpha \beta C}=0, \quad \forall \alpha \neq \beta$,

(II.1) $\quad \omega_{1 \alpha}=\frac{1}{\lambda-\mu}\left(\mu_{1} \omega_{\alpha}+h_{1 \alpha n} \omega_{n}\right)$,

$$
\omega_{1 n}=\frac{1}{\lambda-\sigma}\left(\lambda_{n} \omega_{1}+\sum_{\beta} h_{1 \beta n} \omega_{\beta}+\sigma_{1} \omega_{n}\right) \text {, }
$$

$$
\omega_{\alpha n}=\frac{1}{\mu-\sigma}\left(h_{1 \alpha n} \omega_{1}+\mu_{n} \omega_{\alpha}\right) \text {. }
$$

Recall that the curvature tensor of $M^{n}$ is given by $R_{A B C D}=\delta_{A C} \delta_{B D}$ $-\delta_{A D} \delta_{B C}+h_{A C} h_{B D}-h_{A D} h_{B C}$.

Differentiating (II.3) and applying equations (II.1)-(II.3) and the structure equations of $M^{n}$ to the resulting equation, we compute

$$
\begin{aligned}
\mathrm{LHS}= & d \omega_{\alpha n}=\omega_{\alpha 1} \wedge \omega_{1 n}+\sum_{\beta} \omega_{\alpha \beta} \wedge \omega_{\beta n}-(1+\mu \sigma) \omega_{\alpha} \wedge \omega_{n} \\
= & -\frac{1}{\lambda-\mu}\left(\mu_{1} \omega_{\alpha}+h_{1 \alpha n} \omega_{n}\right) \\
& \wedge \frac{1}{\lambda-\sigma}\left(\lambda_{n} \omega_{1}+\sum_{\beta} h_{1 \beta n} \omega_{\beta}+\sigma_{1} \omega_{n}\right) \\
& +\sum_{\beta} \omega_{\alpha \beta} \wedge \frac{1}{\mu-\sigma}\left(h_{1 \beta n} \omega_{1}+\mu_{n} \omega_{\beta}\right)-(1+\mu \sigma) \omega_{\alpha} \wedge \omega_{n},
\end{aligned}
$$




$$
\begin{aligned}
\text { RHS }= & \left(d \frac{1}{\mu-\sigma}\right) \wedge\left(h_{1 \alpha n} \omega_{1}+\mu_{n} \omega_{\alpha}\right) \\
& +\frac{1}{\mu-\sigma}\left(d h_{1 \alpha n} \wedge \omega_{1}+h_{1 \alpha n} d \omega_{1}+d \mu_{n} \wedge \omega_{\alpha}+\mu_{n} d \omega_{\alpha}\right) \\
= & \left(d \frac{1}{\mu-\sigma}\right) \wedge\left(h_{1 \alpha n} \omega_{1}+\mu_{n} \omega_{\alpha}\right) \\
& +\frac{1}{\mu-\sigma}\left[d h_{1 \alpha n} \wedge \omega_{1}+h_{1 \alpha n}\left(\sum_{\beta} \omega_{1 \beta} \wedge \omega_{\beta}+\omega_{1 n} \wedge \omega_{n}\right)\right.
\end{aligned}
$$$$
+d \mu_{n} \wedge \omega_{\alpha}
$$$$
\left.+\mu_{n}\left(\omega_{\alpha 1} \wedge \omega_{1}+\sum_{\beta} \omega_{\alpha \beta} \wedge \omega_{\beta}+\omega_{\alpha n} \wedge \omega_{n}\right)\right] .
$$

Picking up only those terms of the type of $\omega_{\beta} \wedge \omega_{n}$, we get

$$
\begin{aligned}
\text { LHS }= & -\frac{1}{(\lambda-\mu)(\lambda-\sigma)}\left(\mu_{1} \omega_{\alpha} \wedge \sigma_{1} \omega_{n}+h_{1 \alpha n} \omega_{n} \wedge \sum_{\beta} h_{1 \beta n} \omega_{\beta}\right) \\
& -(1+\mu \sigma) \omega_{\alpha} \wedge \omega_{n}, \\
\text { RHS }= & -\frac{\mu_{n}-\sigma_{n}}{(\mu-\sigma)^{2}} \omega_{n} \wedge \mu_{n} \omega_{\alpha} \\
& +\frac{1}{\mu-\sigma}\left[h_{1 \alpha n}\left(\sum_{\beta} \frac{h_{1 \beta n}}{\lambda-\mu} \omega_{n} \wedge \omega_{\beta}+\sum_{\beta} \frac{h_{1 \beta n}}{\lambda-\sigma} \omega_{\beta} \wedge \omega_{n}\right)\right. \\
& \left.\quad+\left(\mu_{n n} \omega_{n} \wedge \omega_{\alpha}+\frac{\mu_{n}}{\mu-\sigma} \omega_{\alpha} \wedge \omega_{n}\right)\right] .
\end{aligned}
$$

Compare the coefficients of $\omega_{\beta} \wedge \omega_{n}$ and note that $-\frac{1}{(\mu-\sigma)(\lambda-\mu)}+$ $\frac{1}{(\mu-\sigma)(\lambda-\sigma)}=-\frac{1}{(\lambda-\mu)(\lambda-\sigma)}$, we find $\forall \alpha, \beta$,

$$
\begin{aligned}
& -\frac{\mu_{1} \sigma_{1}}{(\lambda-\mu)(\lambda-\sigma)} \delta_{\alpha \beta}+\frac{h_{1 \alpha n} h_{1 \beta n}}{(\lambda-\mu)(\lambda-\sigma)}-(1+\mu \sigma) \delta_{\alpha \beta} \\
& \quad=\frac{\mu_{n}\left(\mu_{n}-\sigma_{n}\right)}{(\mu-\sigma)^{2}} \delta_{\alpha \beta}-\frac{h_{1 \alpha n} h_{1 \beta n}}{(\lambda-\mu)(\lambda-\sigma)}-\frac{\mu_{n n}}{\mu-\sigma} \delta_{\alpha \beta}+\frac{\mu_{n}}{(\mu-\sigma)^{2}} \delta_{\alpha \beta} .
\end{aligned}
$$

Hence

$$
2 h_{1 \alpha n} h_{1 \beta n}=z \delta_{\alpha \beta}
$$


where $z$ is a smooth function on $M^{n}$ defined as

$$
\begin{aligned}
z= & (1+\mu \sigma)(\lambda-\mu)(\lambda-\sigma)+\mu_{1} \sigma_{1}+\frac{\mu_{n}\left(\mu_{n}-\sigma_{n}\right)}{(\mu-\sigma)^{2}}(\lambda-\mu)(\lambda-\sigma) \\
& -\frac{\mu_{n n}}{\mu-\sigma}(\lambda-\mu)(\lambda-\sigma)+\frac{\mu_{n}}{(\mu-\sigma)^{2}}(\lambda-\mu)(\lambda-\sigma) .
\end{aligned}
$$

Let $Y^{t}$ denote the transpose of $Y$. In the form of matrix, the above equation reads as

$$
Y^{t} Y=\frac{1}{2} z I_{q}
$$

Since $Y \in M_{1 \times q}$ with $q \geq 2$, it follows that $Y=0$ everywhere as desired.

Case 2. $p \geq 2$.

Arguing as before, we further have

$$
\lambda_{i}=\mu_{i}=\sigma_{i}=0, \quad \forall i=1, \ldots, p
$$

And equations (2.1)-(2.3) now read as

$$
\begin{aligned}
\omega_{i \alpha} & =\frac{1}{\lambda-\mu} h_{i \alpha n} \omega_{n}, \\
\omega_{i n} & =\frac{1}{\lambda-\sigma}\left(\lambda_{n} \omega_{i}+\sum_{\beta} h_{i \beta n} \omega_{\beta}\right), \\
\omega_{\alpha n} & =\frac{1}{\mu-\sigma}\left(\sum_{j} h_{j \alpha n} \omega_{j}+\mu_{n} \omega_{\alpha}\right) .
\end{aligned}
$$

Similarly, by differentiating (II.1) we have

$$
\begin{aligned}
\mathrm{LHS}= & \sum_{j} \omega_{i j} \wedge \omega_{j \alpha}+\sum_{\beta} \omega_{i \beta} \wedge \omega_{\beta \alpha}+\omega_{i n} \wedge \omega_{n \alpha}-(1+\lambda \mu) \omega_{i} \wedge \omega_{\alpha} \\
& \sim \frac{1}{\lambda-\sigma}\left(\lambda_{n} \omega_{i}+\sum_{\beta} h_{i \beta n} \omega_{\beta}\right) \wedge\left(-\frac{1}{\mu-\sigma}\right)\left(\mu_{n} \omega_{\alpha}+\sum_{j} h_{j \alpha n} \omega_{j}\right) \\
& -(1+\lambda \mu) \omega_{i} \wedge \omega_{\alpha},
\end{aligned}
$$




$$
\begin{aligned}
\mathrm{RHS} & =\left(d \frac{1}{\lambda-\mu}\right) \wedge h_{i \alpha n} \omega_{n} \\
& +\frac{1}{\lambda-\mu}\left[d h_{i \alpha n} \wedge \omega_{n}+h_{i \alpha n}\left(\sum_{j} \omega_{n j} \wedge \omega_{j}+\sum_{\beta} \omega_{n \beta} \wedge \omega_{\beta}\right)\right] \\
& \sim \frac{h_{i \alpha n}}{\lambda-\mu}\left(-\frac{1}{\lambda-\sigma} \sum_{j, \beta} h_{j \beta n} \omega_{\beta} \wedge \omega_{j}-\frac{1}{\mu-\sigma} \sum_{j, \beta} h_{j \beta n} \omega_{j} \wedge \omega_{\beta}\right) \\
& =\sum_{j, \beta} \frac{h_{i \alpha n} h_{j \beta n}}{\lambda-\mu}\left(\frac{1}{\lambda-\sigma}-\frac{1}{\mu-\sigma}\right) \omega_{j} \wedge \omega_{\beta} \\
& =-\sum_{j, \beta} \frac{h_{i \alpha n} h_{j \beta n}}{(\lambda-\sigma)(\mu-\sigma)} \omega_{j} \wedge \omega_{\beta},
\end{aligned}
$$

where for any two given 2-forms $\psi$ and $\psi^{\prime}$, by $\psi \sim \psi^{\prime}$ we mean $\psi \equiv \psi^{\prime}\left(\bmod \omega_{n}\right)$, i.e., $\psi-\psi^{\prime}=\omega \wedge \omega_{n}$ for some 1 -form $\omega$.

Now, by picking up those terms of the type of $\omega_{j} \wedge \omega_{\beta}$ we have

$$
\begin{aligned}
& -\frac{1}{(\lambda-\sigma)(\mu-\sigma)}\left(\lambda_{n} \omega_{i} \wedge \mu_{n} \omega_{\alpha}+\sum_{\beta} h_{i \beta n} \wedge \sum_{j} h_{j \alpha n} \omega_{j}\right) \\
& -(1+\lambda \mu) \omega_{i} \wedge \omega_{\alpha} \\
& \quad=-\sum_{j, \beta} \frac{h_{i \alpha n} h_{j \beta n}}{(\lambda-\sigma)(\mu-\sigma)} \omega_{j} \wedge \omega_{\beta} .
\end{aligned}
$$

Then,

$$
2 h_{i \alpha n} h_{j \beta n}=\bar{z} \delta_{i j} \delta_{\alpha \beta}, \quad \forall i, j, \alpha, \beta
$$

where $\bar{z}=\lambda_{n} \mu_{n}+(1+\lambda \mu)(\lambda-\sigma)(\mu-\sigma)$.

In particular,

$$
h_{i \alpha n} h_{i \beta n}=\frac{1}{2} \bar{z} \delta_{\alpha \beta}, \quad \forall i, \alpha, \beta
$$

Again, since $q \geq 2$ we have $h_{i \alpha n}=0, \forall i, \alpha$, i.e. $Y=0$ everywhere.

This establishes the Key Lemma and thus completes the proof of the Main Theorem. 


\section{REFERENCES}

[1] R. Bryant, private conversation.

[2] E. Cartan, Sur des familles remarquables d'hypersurfaces isoparametriques dans les espaces spheriques, Math. Z., 45 (1939), 335-367.

[3] S. Chang, On minimal hypersurfaces with constant scalar curvatures in $S^{4}$, J. Differential Geom., 37 (1993), 523-534.

[4] _ A closed hypersurface of constant scalar curvature and constant mean curvature in $S^{4}$ is isoparametric, Comm. in Analysis and Geometry, 1 (1993), 71-100.

[5] S. S. Chern, M. do Carmo and S. Kobayashi, Minimal submanifolds of a sphere with second fundamental form of constant length, Functional analysis and related fields, Springer, Berlin, Heidelberg, New York, 1970, pp. 59-75.

[6] H. B. Lawson, Local rigidity theorems for minimal hypersurfaces, Ann. of Math., 89 (1969), 187-191.

[7] R. Miyaoka, Complete hypersurfaces in the space form with three principal curvatures, Math. Z., 179 (1982), 345-354.

[8] T. Otsuki, Minimal hypersurfaces in a Riemannian manifold of constant curvature, Amer. J. Math., 92 (1970), 145-173.

[9] C. K. Peng and C. L. Terng, Minimal hypersurface of spheres with constant scalar curvature, Ann. of Math. Stud., No. 103 (1983), 177-198.

[10] _ The scalar curvature of minimal hypersurfaces in spheres, Math. Ann., 266 (1983), 105-113.

[11] J. Simons, Minimal varieties in a Riemannian manifold, Ann. of Math., 88 (1968), 62-105.

[12] S. T. Yau, Problem section, Ann. of Math. Stud., No. 102 (1982), 693.

Received June 2, 1992.

UNIVERSITY OF UTAH

SALT LAKe City, UT 84112-1107 


\section{PACIFIC JOURNAL OF MATHEMATICS}

Founded by

E. F. BECKENBACH (1906-1982) F. Wolf (1904-1989)

\section{EDITORS}

Sun-Yung A. Chang

(Managing Editor)

University of California

Los Angeles, CA 90024-1555

chang@math.ucla.edu

\section{F. Michael Christ}

University of California

Los Angeles, CA 90024-1555

christ@math.ucla.edu

Herbert Clemens

University of Utah

Salt Lake City, UT 84112

clemens@math.utah.edu

\author{
ThOMAs ENRIGHT \\ University of California, San Diego \\ La Jolla, CA 92093 \\ tenright@ucsd.edu \\ Nicholas ERcolaNI \\ University of Arizona \\ Tucson, AZ 85721 \\ ercolani@math.arizona.edu \\ R. FINN \\ Stanford University \\ Stanford, CA 94305 \\ finn@gauss.stanford.edu \\ VAUGHAN F. R. Jones \\ University of California \\ Berkeley, CA 94720 \\ vfr@math.berkeley.edu
}

\author{
STEVEn KerCKHOFF \\ Stanford University \\ Stanford, CA 94305 \\ spk@gauss.stanford.edu
}

Martin ScharlemanN

University of California

Santa Barbara, CA 93106

mgscharl@math.ucsb.edu

\author{
Harold Stark \\ University of California, San Diego \\ La Jolla, CA 92093 \\ V. S. VARADARAJAN \\ University of California \\ Los Angeles, CA 90024-1555 \\ vsv@math.ucla.edu
}

\section{SUPPORTING INSTITUTIONS}

UNIVERSITY OF ARIZONA

UNIVERSITY OF BRITISH COLUMBIA

CALIFORNIA INSTITUTE OF TECHNOLOGY

UNIVERSITY OF CALIFORNIA

UNIVERSITY OF MONTANA

UNIVERSITY OF NEVADA, RENO

NEW MEXICO STATE UNIVERSITY

OREGON STATE UNIVERSITY

\author{
UNIVERSITY OF OREGON \\ UNIVERSITY OF SOUTHERN CALIFORNIA \\ STANFORD UNIVERSITY \\ UNIVERSITY OF HAWAII \\ UNIVERSITY OF UTAH \\ WASHINGTON STATE UNIVERSITY \\ UNIVERSITY OF WASHINGTON
}

The Supporting Institutions listed above contribute to the cost of publication of this Journal, but they are not owners or publishers and have no responsibility for its content or policies.

Mathematical papers intended for publication in the Pacific Journal of Mathematics should be in typed form or offset-reproduced (not dittoed), double spaced with large margins. Please do not use built up fractions in the text of the manuscript. However, you may use them in the displayed equations. Underline Greek letters in red, German in green, and script in blue. The first paragraph must be capable of being used separately as a synopsis of the entire paper. In particular it should contain no bibliographic references. Please propose a heading for the odd numbered pages of less than 35 characters. Manuscripts, in triplicate, may be sent to any one of the editors. Please classify according to the 1991 Mathematics Subject Classification scheme which can be found in the December index volumes of Mathematical Reviews. Supply name and address of author to whom proofs should be sent. All other communications should be addressed to the managing editor, or Julie Honig, University of California, Los Angeles, California 90024-1555.

There are page-charges associated with articles appearing in the Pacific Journal of Mathematics. These charges are expected to be paid by the author's University, Government Agency or Company. If the author or authors do not have access to such Institutional support these charges are waived. Single authors will receive 75 free reprints; joint authors will receive a total of 100 free reprints. Additional copies may be obtained at cost in multiples of 50 .

The Pacific Journal of Mathematics (ISSN 0030-8730) is published monthly except for July and August. Regular subscription rate: $\$ 215.00$ a year (10 issues). Special rate: $\$ 108.00$ a year to individual members of supporting institutions.

Subscriptions, orders for numbers issued in the last three calendar years, and changes of address should be sent to Pacific Journal of Mathematics, P.O. Box 4163, Berkeley, CA 94704-0163, U.S.A. Old back numbers obtainable from Kraus Periodicals Co., Route 100, Millwood, NY 10546.

The Pacific Journal of Mathematics at University of California, c/o Department of Mathematics, 981 Evans Hall, Berkeley, CA 94720 (ISSN 0030-8730) is published monthly except for July and August. Second-class postage paid at Berkeley, CA 94704, and additional mailing offices. POSTMASTER: send address changes to Pacific Journal of Mathematics, P.O. Box 4163, Berkeley, CA 94704-0163.

PUBLISHED BY PACIFIC JOURNAL OF MATHEMATICS at University of California, Berkeley, CA 94720, A NON-PROFIT CORPORATION

This publication was typeset using $\mathcal{A} \mathcal{M} \mathcal{S}-\mathrm{T}_{\mathrm{E}} \mathrm{X}$,

the American Mathematical Society's $T_{E} X$ macro system.

Copyright (c) 1994 by Pacific Journal of Mathematics 


\section{PACIFIC JOURNAL OF MATHEMATICS}

Volume $165 \quad$ No. $1 \quad$ September 1994

The effect of dimension on certain geometric problems of irregularities of 1 distribution

RALPH ALEXANDER

The structure of $\mathrm{sl}(2,1)$-supersymmetry: irreducible representations and primitive ideals

Didier ARnAL, HÉdi BENAMOR and GEORges PinCZON

Periods and Lefschetz zeta functions

Josefina CASASAYas, JaUme Llibre and ANA Nunes

On closed hypersurfaces of constant scalar curvatures and mean curvatures in 67 $S^{n+1}$

\section{SHAOPING CHANG}

$R$-groups and elliptic representations for $\mathrm{SL}_{n}$

DAVID GOLDBERG

The boundary distortion of a quasiconformal mapping

JuHA HeINONEN and PEKKA KosKela

Strongly approximately transitive group actions, the Choquet-Deny theorem, 115 and polynomial growth

\section{WOJCIECH JAWORSKI}

$q$-canonical commutation relations and stability of the Cuntz algebra

PALle E. T. Jorgensen, L. M. Schmitt and Reinhard Frank

WERNER

Complete open manifolds of non-negative radial curvature

YOSHIROH MACHIGASHIRA

Perturbations of certain reflexive algebras

DAVID RYDER PITTS

Interpolation submanifolds of the unitary group 